\title{
Jets from Galactic Binaries
}

\section{Thomas J. Maccarone*}

Faculty of Applied and Physical Sciences

University of Southampton, Southampton - U.K.

E-mail: tjmesoton.ac.uk

I present a brief review of the properties of jets from X-ray binaries, highlighting the disk-jet connection, in which there are strong correlations between X-ray and radio power for black holes and for neutron star in low/hard spectral states, and reduced emission in soft states. I discuss how some of the new "deviant" black hole systems which follow the relation normally found for neutron stars might fit into such a picture. I close by highlighting a few open questions which might be best addressed with soft gamma-ray observations.

The Extreme and Variable High Energy Sky

September 19-23, 2011

Chia Laguna (Cagliari), Italy

\footnotetext{
*Speaker.
} 


\section{Introduction}

Jets are ubiquitous in astrophysics, being seen from nearly all classes of accreting objects. In this paper, I will briefly review the contributions to our understanding of jets that have come from modelling and observations of Galactic sources, and in the spirit of this conference's aims, will put a bit of emphasis on where future hard X-ray and gamma-ray observations may contribute to futher understanding of jet production and jet physics.

Jets play several important roles in the Universe. They are one of the dominant candidates for providing the feedback of energy into the interstellar medium that stops cooling flows from being found in most clusters of galaxies. Jets are also one of the best candidates for producing the highest energy cosmic rays, which have energies orders of magnitude higher than those which can be produced in particle accelerators. The production of jets may provide key information about the underlying accretion flows that power them, and the jets themselves provide important laboratories for testing plasma physics under extreme conditions.

The viscous timescale from the innermost stable circular orbit in a Shakura-Sunyaev (1973) accretion disk is about 100 years for a $10^{8} M_{\odot}$ black hole - and much larger from the outer part of the disk. The variability in most AGN thus cannot reflect any fundamental change in system parameters. X-ray binaries, on the other hand, can change in luminosity by 6-7 orders of magnitude on timescales of a year or so, allowing one to study how the jet power is affected by accretion rate changes in a single system, so that one does not need to amass a large sample of objects to control for black hole mass or spin. Additionally, X-ray binaries generally are dominated in bolometric luminosity in the X-rays, meaning that it is straightforward to understand how the accretion rate is changing without covering hard-to-observe regions of the spectrum like the far-infrared or ultraviolet. These advantages make it much easier to understand the accretion-ejection connection in $\mathrm{X}$-ray binaries than in any other class of objects.

\section{Spectral states and the disk-jet connection}

X-ray binaries exhibit a range of "spectral state" phenomenology. These states represent collections of properties of the source, with sharp changes in the source spectral energy distributions taking place in concert with sharp changes in the source power spectra. The longest lived states are the low/hard state and the high/soft state. The high/soft state agrees very well with the predictions of the standard geometrically thin, optically thick, thermally emitting accretion disk model of Shakura \& Sunyaev (1973). In the low/hard state, source spectra are reasonably well fitting by cutoff power laws with photon indices of about 1.7 and cutoff energies around $100 \mathrm{keV}$, well explained by thermal comptonization in a geometrically thick, optically thin flow (Thorne \& Price 1975). The sources show strong red-noise variability with fractional rms amplitude of about $30 \%$. Low magnetic field neutron star sources show the same basic phenomenology, but with slightly more complicated spectra, probably related to their having both boundary layers and accretion disks, each of which can contribute quasi-thermal emission. In both classes of sources, the transitions are hysteretical, with the transition from hard to soft usually occurring near the peak of an outburst (Miyamoto et al 1995; Maccarone \& Coppi 2003), and the transition from soft-to-hard states usually occurring near $2 \%$ of the Eddington luminosity (Maccarone 2003). At the state transi- 
tions, hybrid spectra are seen, which tend to have strong thermal and strong power law components simultaneously, and the power spectra often show strong quasi-periodic oscillations.

Two correlations are clearly exhibited between accretion disk properties and jet power (as measured from the radio flux) in black hole X-ray binaries. The first is the switch-off of the radio emission in the high/soft state (Tananbaum et al. 1972; Fender et al. 1999). The standard mechanisms for powering relativistic jets require large scale height magnetic fields (Livio et al. 1999; Meier 2001), so that the radio jet would be expected to become much less powerful when the accretion flow becomes geometrically thin. The recent finding that the radio power of the persistently soft state black hole $4 \mathrm{U} 1957+11$ is at least a factor of 300 below the extrapolation of the hard state relation (Russell et al. 2011) comes close to presenting a challenge to theory.

The other correlation which has been found is one between X-ray luminosity and radio luminosity in the hard state (Hannikainen et al. 1998; Corbel et al. 2000; Gallo, Fender \& Pooley 2003 - GFP). Most hard state black hole X-ray binaries have been found to follow a relation where $L_{R} \propto L_{X}^{0.7}$ (GFP). This relation is consistent with theoretical expectations under three assumptions: (1) the kinetic power into the jet scales linearly with the mass accretion rate onto the black hole (2) the radio power follows standard synchrotron theory for conical, self-absorbed jets, and hence $L_{R} \propto L_{k i n}^{1.4}$ and (3) the accretion onto the black hole is radiatively inefficient, with $L_{X} \propto \dot{m}^{2}$, consistent with expecations from an advection dominated accretion flow (e.g. Narayan \& Yi 1995).

Two key differences present themselves between the radio properties of low magnetic field neutron star X-ray binaries and those of black hole X-ray binaries. The first is that the radio/X-ray correlation in the neutron stars seems to follow the $L_{R} \propto L_{X}^{1.4}$ relation as a rule in the neutron stars (e.g. Migliari et al. 2003 - M03). The second is that the neutron star systems can show relatively strong radio emission in soft states (e.g. Migliari et al. 2004). Both of these differences can be understood as boundary layer effects. The steeper slope for the radio/X-ray relation in the hard state is straightforward to explain - the X-ray emission is expected to be radiatively efficient for accretion onto a neutron star (M03). The radio emission in the soft states can be explained as being powered by the boundary layer itself - the boundary layer is necessarily differentially rotation, and necessarily has a scale height comparable to its distance from the center of the neutron star (e.g. Maccarone 2008). The magnetic field of the neutron star itself may also contribute to jet launching (D. Meier, private communication).

In recent years, a few black hole systems have been seen that are underluminous in the radio relative to the sample studied by GFP. In one of the best studied cases, that of H1743-322, Coriat et al. (2011) showed that the source follows a $L_{R} \propto L_{X}^{1.4}$ relation in the brighter parts of its hard state, before transiting to a more standard $L_{R} \propto L_{X}^{0.7}$ relation deeper into the hard state. The steeper relation has been seen in several other sources in recent years. A possible explanation is that in these "deviant" hard states, the accretion flow is really radiatively efficient. This might be explained if, e.g. there exists a geometrically thin, optically thick accretion disk at the center of the accretion flow - something that has been suggested in another context by Meyer-Hofmeister et al. (2009). Such a disk could be a relic of the high/soft state, since, due to the high density of gas in the innermost regions, the evaporation of the thin disk into a thick, hot disk is expected to happen later in the outburst decay than the evaporation of the disk at moderate distances from the black hole. Since the innermost part of the accretion flow would be a thin disk, one would then expect that such a flow would be radiatively efficient - and, in fact, much like the neutron star accretion 
flows in the hard state which join to boundary layers in their centers. It is worth noting that the radio weak hard states have been seen primarily in X-ray binaries which fade very slowly from their peak brightnesses, and that this slow fading may be related to their inability to evaporate their entire inner disks into hot, thick accretion flows.

\section{Short wavelength emission from jets}

In most cases, in the low hard states, the emission region size in the radio will be many light minutes across or more, limiting the amount of rapid variability that can be expected. As a result, to look for the most rapid variability from the jet, and hence to probe how it is powered on short timescales, it is necessary to look at higher frequency photons, which come from closer in to the black hole or neutron star. The development of high-speed solid state photometers in the optical and infrared have made such measurements possible in recent years, after decades in which relatively little high speed work had been done in this wavelength range. The optical data have been puzzling, typically showing both a positively correlated component in the cross-correlation with a moderate time lag, and an anti-correlated component, often at negative time lag. This result has been explained in terms of a magnetic energy reservoir model in which the optical emission comes from the jet (Malzac et al. 2004), and in terms of a model in which the optical emission comes from a combination of the synchrotron emission from the corona whose Compton upscattering produces the X-rays, and some thermal reprocessing in the outer accretion disk (Veledina et al. 2011).

At the present time, only one example of sub-second variability in the infrared has been presented in the literature (Casella et al. 2010). It is a much simpler observation, with the infrared emission lagging behind the $\mathrm{X}$-rays by about 0.1 seconds, but with no statistically significant anticorrelations and with no strong asymmetries to the cross-correlation function. Additionally, the brightness temperature of the infrared emission is sufficiently high that it cannot be thermal emission without the expectation that the thermal X-ray emission from that component would exceed the observed X-ray flux. As a result, in this case, the infrared emission must really be coming from the relativistic jet that produced the radio emission, and the time delay is likely revealing the travel time of perturbations to the region where the infrared emission is produced, making IR variability a potentially powerful tool for mapping out how jets are powered.

It is clear that jets may emit detectably at high energies when they collide with the interstellar medium(e.g. Corbel et al. 2002), or the stellar winds of their systems' donor stars (e.g. Tavani et al. 2009; Fermi LAT Collaboration 2009). On the other hand, the more interesting question concerns the viability of models which suggest that the bulk of the hard X-ray emission from "normal" low/hard state systems may come from their jets (e.g. Markoff et al. 2001).

A few pieces of observational evidence indicate that the X-rays do not, in fact, come from the jet. In the neutron star X-ray binary $4 \mathrm{U} 0614+091$, the break from an optically thick compact jet to an optically thin jet can be clearly seen in its broadband spectral energy distribution, and the extrapolation of the optically thin jet falls far below the X-ray flux (Migliari et al. 2010). Additionally, because jets are radiatively efficient, and cannot carry away too large a fraction of the total accretion power, one would expect a sharp drop in the X-ray luminosity at the state change from the high/soft state to low/hard state if the jet were the dominant source of X-ray luminosity in the low/hard state, but sharp transitions are not seen (Maccarone 2005). Therefore, it seems most 
likely that the dominant mechanism for producing the X-rays in the hard states of X-ray binaries is, in fact, thermal Comptonization, and that the correlation between X-rays and radio is simply a result of the higher power to the jet expected at high accretion rate even in the context of a standard underlying thin or thick accretion disk (e.g. Meier 2001).

\section{Do the black hole spins matter?}

Many recent attempts have been made to measure black hole spins in the past decade or so. The two most-used methods are reflection model fitting (e.g. Ross \& Fabian 2005) and thermal continuum fitting (e.g. Davis \& Hubeny 2006). Attempts to correlate either the radio power, or the ratio of the radio power to the X-ray power with these spin indicators have not revealed any detectable correlations, indicating that the black hole spin measurements are in error, or that the jet power does not correlate strongly with the black hole spin, or both (Fender, Gallo, Russell 2010). This result lies in constrast with some claims from studies of active galactic nuclei, which find correlations between radio power and properties of their host galaxies suggested to correlate with black hole merger history, and hence spin (Balmaverde \& Capetti 2006; Sikora et al. 2007); however, both of these results neglect to account for the effects of the black hole mass on the radio flux (e.g. Merloni et al. 2003), and furthermore, the latter of these papers fails to deal properly with extended emission which may be resolved out for some objects and not others. There is thus still no strong observational evidence that black hole spin is important for powering jets, however strong the theoretical considerations for it may be.

\section{Some key open questions}

As the purpose of this meeting was partly to serve as the 9th birthday party for INTEGRAL, I highlight a few open questions in jet physics that might best be addressed with INTEGRAL. One is the aforementioned role of the neutron star's magnetic field, which would be best studied by getting increased sample sizes of millisecond X-ray pulsars - a class of objects INTEGRAL has been especially successful in discovering. A second is an understanding of the effects of spectral state transition hysteresis on jet properties - again something with which INTEGRAL can help, since its harder response and better sensitivity relative to other wide field monitors makes it more sensitive to early activity of X-ray transients. An additional one is the pair composition of jets Granat tenatively detected a redshifted annihilation line from Nova Muscae near state transition (Gilfanov et al. 1991), but the far more sensitive INTEGRAL still has not been able to observe an $\mathrm{X}$-ray transient in a similar spectra state.

\section{References}

[1] Balmaverde B., Capetti A., 2006, A\&A, 447, 97

[2] Casella P., et al., 2010, MNRAS, 404L, 21

[3] Corbel S., Fender R.P., Tzioumis A.K., Nowak M.A., McIntyre V., Durouchoux P., Sood R., 2000, A\&A, 359, 251 
[4] Corbel S., Fender R.P., Tzioumis A.K., Tomsick J.A., Orosz J.A., Miller J.M., Wijnands R., Kaaret P., 2002, Science, 298, 196

[5] Coriat M., et al., 2011, MNRAS, 414, 677

[6] Davis S.W. \& Hubeny I., 2006, ApJS, 164, 530

[7] Fender R.P., et al., 1999, ApJ, 519L, 165

[8] Fender R.P., Gallo E., Russell D., 2010, MNRAS, 406, 1425

[9] Fermi LAT Collaboration, 2009, Science, 326, 1512

[10] Gallo E., Fender R.P., Pooley G.G., 2003, MNRAS, 344, 60

[11] gilfanov M., et al., 1991, SvAL, 17, 437

[12] Hannikainen D.C., Hunstead R.W., Campbell-Wilson D., Sood R.K., 1998, A\&A, 337, 460

[13] Livio M., Ogilvie G.I., Pringle J.E., 1999, ApJ, 512, 100

[14] Maccarone T.J., 2005, MNRAS, 360L, 68

[15] Markoff S., Falcke H., Fender R., 2001, A\&A, 372L, 25

[16] Meier D.L., 2001, ApJ, 548L, 9

[17] Merloni A., Heinz S., Di Matteo T., 2003, MNRAS, 345, 1057

[18] Migliari S., Fender R.P., Rupen M., Jonker P.G., Klein-Wolt M., Hjellming R.M., van der Klis M., 2003, MNRAS, 342L, 67

[19] Migliari S., Fender R.P., Rupen M., Wachter S., Jonker P.G., Homan J., van der Klis M., 2004, MNRAS, 351, 186

[20] Maccarone T.J., 2003, A\&A, 409, 697

[21] Maccarone T.J., Coppi P.S., 2003, MNRAS, 338, 189

[22] Maccarone T.J., 2008, ASPC, 401, 191

[23] Malzac J., Merloni A., Fabian A.C., 2004, MNRAS, 351, 253

[24] Meyer-Hofmeister E., Liu, B.F., Meyer F., 2009, A\&A, 508, 329

[25] Migliari S., et al., 2010, ApJ, 710, 117

[26] Miyamoto S., Kitamoto S., Hayashida K., Egoshi W., 1995, ApJ, 442L, 13

[27] Narayan R., Yi, I., 1995, ApJ, 452, 710

[28] Ross R.R., Fabian A.C., 2005, MNRAS, 358, 211

[29] Shakura N.I., Sunyaev R.A., 1973,A\&A, 24, 337

[30] Sikora M., Stawarz L., Lasota J.-P., 2007, ApJ, 658, 815

[31] Tananbaum H., Gursky H., Hellogg E., Giacconi R., Jones C., 1972, ApJ, 177L, 5

[32] Tavani M., et al., 2009, Nature, 462, 620

[33] Thorne K.S., Price R.H., 1975, ApJ, 195L, 101

[34] Veledina A., Poutanen J., Vurm I., 2011, ApJ, 737L, 17 